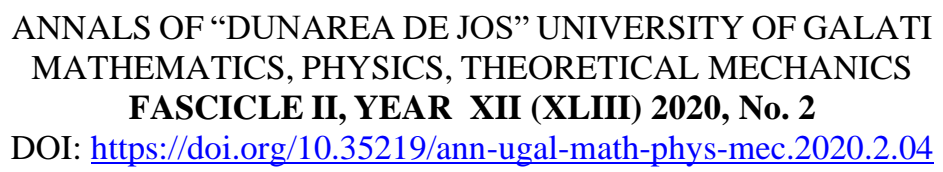

\title{
Advances in education through IoT
}

\author{
Daniel Ganea ${ }^{1}$, Marian Craciun $^{1}$, Catalin Arama ${ }^{1}$, Ciprian Vlad $^{1 *}$

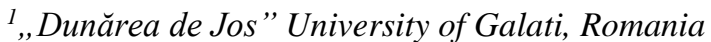 \\ *Correspondence: Ciprian.Vlad@ugal.ro
}

\begin{abstract}
Context: The education sector is the root of our society. Living in a fast changing environment, we need to keep pace by implementing new technologies and techniques. A widespread solution is the Internet of Things together with Cloud computing technologies. Both technologies offer sustainable solutions for improving the efficiency of learning and teaching. Thus, these IT solutions become the main aspect of our society by interconnecting heterogeneous devices and technologies in different aspects of life.

Objective: This paper focuses on the Internet of Things' major technologies and applications with an orientation in the education sector. This overview can help scholars and academia to adopt and participate in the endeavor to streamline the receive - transmit process of education.

Method: A systematic literature review was performed considering the main scientific databases. The number of the research paper was narrowed after the exclusion criteria were applied.

Results: This paper underlines the importance of adopting both IoT and Cloud computing in teaching and learning activities. The evidence on the diversity of both IoT and Cloud computing applications are underline
\end{abstract}

Keywords: IoT, automation, education sector, smart classroom.

\section{INTRODUCTION}

In economics, there is a concept called primary resources. Besides capital and nature, this category also includes human resources. In our society, human resource is the root of any industry or sector. Therefore, regardless of the level of development of any country, much more attention must be granted to educating this resource. The educational process does not only include the volume and quality of information but also the methods used in this process that can have a major impact on transferring and receiving (TX-RX) the data. It can be said that this process is a two-way channel in close connection with both the medium (methods and techniques) and the data.

An increasingly common expression is that we live in a constantly changing world, both environment and people. Thereby, to make the learning and teaching process more efficient, the latest IT solutions should be adopted.

Not long ago, both IoT and Cloud computing gained the attention of the educational system to develop and implement not only smart campus but also smart classrooms [1]. Therefore, around the world, many universities develop a solution using state of the art technologies to provide better services that are efficient, secure, sustainable, and eco-friendly [2-4]. The Internet of Things put classrooms and campus devices on the local area network (LAN). This connectivity brings teachers and students closer by sharing the infrastructure and data in a smart way [1].

The aim of this paper is to describe the efficiency of using IoT in living and working with an orientation to the educational sector. Both IoT and Cloud computing can revolutionize learning methods and traditional education. The pro and cons of implementing the IoT concept in education are also presented in this paper. 


\section{INTERNET OF THINGS AND ITS APPLICATION DOMAINS}

Internet of Things is a concept that evolves through time. Before its development, some predefined periods can be resumed (figure 1):

- Pre-Internet era: This consisted of human-to-human communication through Short Message Service (SMS) or fixed telephonic line;

- Internet of Content: This era is associated with the evolution of the World Wide Web (WWW). This is also where the foundations were laid for E-mail and MMS (minor messaging service);

- Internet of Services era: This consisted of the evolution of WEB 2.0. In this era, internet functionality has increased and people started to use it more frequently for communication and shopping. This is the era where E-Commerce and E-productivity has emerged;

- Internet of People: In this era, people are more connected with one another. Services like, Twitter, Skype, YouTube, Facebook, LinkedIn, etc. were created;

- Internet of Things: this is the era of machine-to-machine communication minimizing human interaction.

The concept of the Internet of Things (IoT) refers to a system of interconnected devices and objects. Under this umbrella, each device has a unique identifier (UIDs) and therefore the ability to transfer information over a network without human-to-human or interaction or even human-tocomputer. Nowadays, there is no doubt that the Internet of Things (IoT) offers a new dimension of living or working. According to a recent study [1], until 2021, the number of devices that are interconnected will exponentially grow to about 34 billion, with wide implications in all domains. The aim of the IoT is to create smart and self-aware environments [5]. Nowadays, the concept of IoT reached all aspects of our living and working. To name a few:

- Industrial IoT: The IIoT represents a high number of industrial systems that are connected in order to communicate and coordinate their actions to increase process efficiency. In IIoT there is a strong emphasis on machine learning, machine-to-machine communication and coordination, and big data $[6,7]$;

- $\quad$ IoT in smart cities: The IoT concepts implemented in urban and rural areas increases the quality of life. This include [8-11]: sustainable water supply, city lighting and traffic monitoring, garbage management, citizens safety and crowdsensing, air standard and environment, smart parking, public transportation, etc.;

- IoT in energy domain: The EIoT offers flexibility to adapt the new energy sources, improved management of both operations and assets, customer service orientation, the possibility of enabling new business models and services, reliability and security [12, 13];

- IoT in the smart home domain: A smart home is composed of sensors, devices, and systems. These components can be controlled and monitored via remote access. There is a variety of smart "objects" in a smart home: fitness trackers, cell phones, water quality sensors, smart bulbs, smart refrigerators and dishwashers, smart cooking devices, etc. IoT implemented in a living environment has numerous advantages due to the fact that minimizes human involvement [14-16].

- $\quad$ IoT in the healthcare domain (IoHT). The IoHT helps to overcome limitations in personnel, financial, and resources. Thus, specialists can monitor and assist even patients living long distances from health care resources $[17,18]$. 


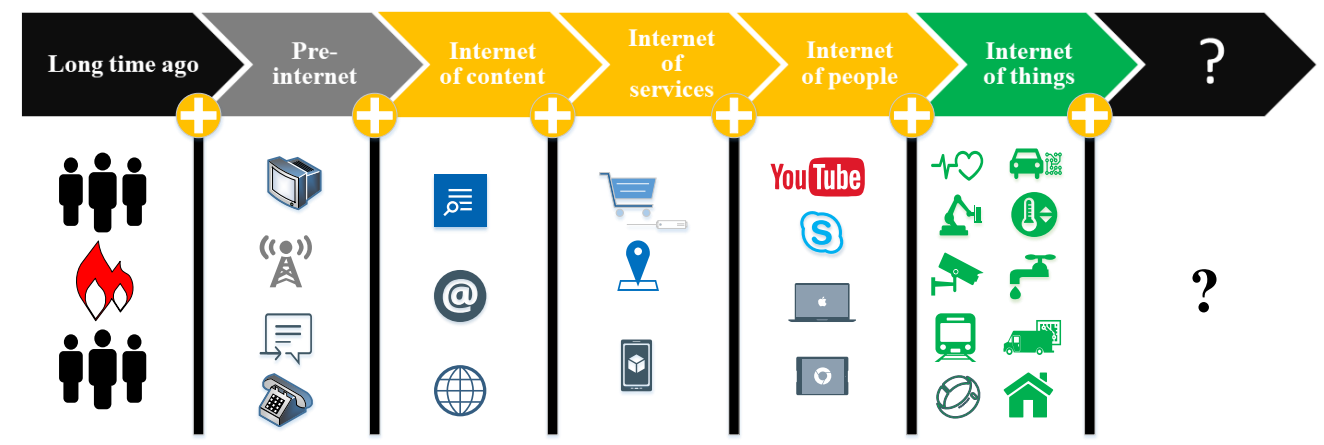

Fig. 1. The evolution of IoT

\section{INTERNET OF THINGS PLATFORM}

In general, the IoT is perceived as an ecosystem. This widely accepted technology enables the interconnection of devices and objects to communicate with each other. This system has a multilayered architecture (figure 2). This consists of the three main layers: the sensory layer (hardware or perception layer), the network layer (communication layer), and the application layer (business layer).

The lowest layer is the sensory layer. Its scope is to interact with the real world by collecting and transferring data to the above layer (network layer) [2,19]. The sensory layer can contain tags, beacons, sensors, health and fitness devices, consumer electronics, automotive, embedded hardware, etc. The main component of this ecosystem is the IoT platform (network layer), an essential component, that connects and supports all ecosystem components. More precisely, this component is a multi-layer technology that offers provisioning, automation, and management to both application and sensory levels $[2,20]$. The network layer is responsible for routing data to the ecosystem devices. The ecosystem devices can be heterogeneous using various communication interfaces and protocols [20, 21]. The thirds layer is the application layer. This layer is at the top of the architecture and is responsible for the analysis and storage of the data sent by the network layer. Its main job is to provide services based on ecosystem requirements. The application layer may contain data storage and analytics capabilities, customer applications, industrial applications, business applications, etc. [2224].

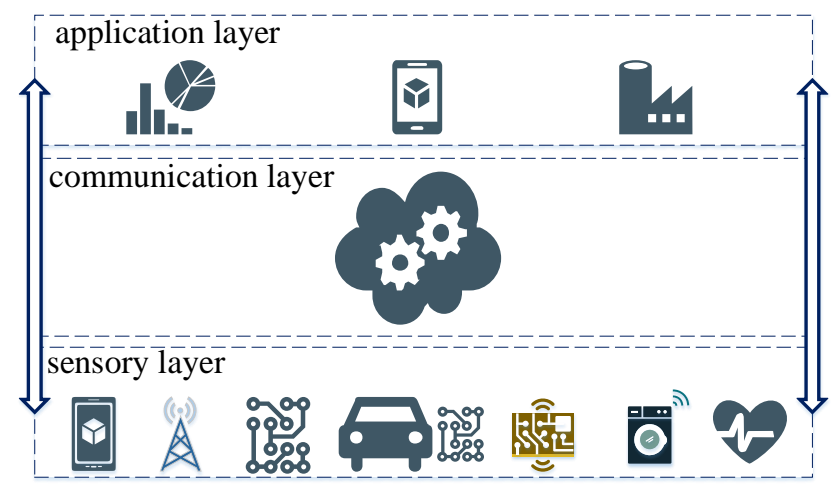

Fig. 2. The IoT layers

\section{IoT IN EDUCATION DOMAIN}

As previously presented, IoT "invaded" all aspects of our living and working. Recently, this technology was applied to one of the most important sectors of our society. This is the educational sector. Applying this technology to a traditional campus or classroom has implications in transforming the RX-TX process of education. 
In a recent study [12], the authors developed a system for optimizing classroom and campus efficiently. The proposed system has the capabilities of determining class occupation for the campus lecture halls. In addition to this, the system has multiple features. One of that is the use of artificial intelligence (AI) for attendance prediction by collecting live occupancy.

Smart campus/classroom teaching platform needs reliable and fast connectivity. One solution is a network based on 5G technology. Xu et al [25] propose a system for monitoring classroom presence by analyzing student location.

Faritha Banu et al [26] proposed a methodology for integrating IoT base Cloud in a smart classroom for a sustainable and smart campus. Through this system, any campus can have multiple benefits: scientific research, attendance monitoring, management of students and staff, finding objects, online billing, and security.

A system that is easy to use and both low power and cost was developed by Zhu et al [27]. The main capabilities of this system are the control of the electrical devices to minimize energy consumption, monitor the environment of the campus and classroom. The proposed management system is based on IoT and a suite of Shanghai Qixiang Technology Co., Ltd sensors.

The root of a smart campus is a smart classroom. Therefore, researcher and developers must orientate their focus and action mainly in optimizing this component. Learning managing system (LMS) is one solution. A Learning Management System (LMS) is a platform or a website designed to streamline the upload process for people with limited technical knowledge. The LMS (figure 3) has a huge potential due to its common features: security, payments, content, scheduling, reporting, tracking and auditing, assessments, certification, communication, marketing and social media, mobile learning, multi-lingual support, etc. All of this boils down to two major benefits: better understanding and lowmaintenance [1].

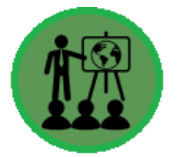

efficiency

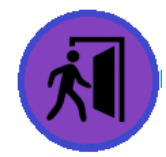

accessibility

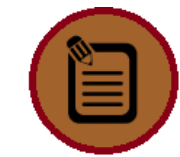

evolving content

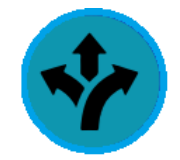

flexibility

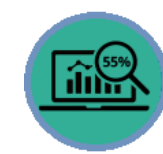

reporting and analytics

Fig. 3. The main components of LMS

\section{CONCLUSIONS}

The Internet of Things is a sustainable and promising paradigm due to the exponentially growing number of "things" that can be connected. Managing these devices may still be considered a challenge but this aspect is shaded by a large number of benefits. The benefits (table 1) obtained by the educational system that may be underline are improved delivery quality of information to students to meet their expectations and needs not only in short-term but also in long-term, quality improvement, production time, reduction of development costs and risks. Such outcomes are generated to the correct researching, planning, and application of both organizational and technical activities. By using IoT in campus and classrooms faculty and management can focus better on their main task and responsibilities. Besides these, IoT in education provides both intelligent and environmentally sustainable education.

Table 1. Pro and Cons of IoT in education.

\begin{tabular}{ll}
\hline \multicolumn{1}{c}{ Pros of IoT } & \multicolumn{1}{c}{ Cons of IoT } \\
\hline cost savings & over dependency on technology \\
\hline Information and communication & losing security on privacy \\
\hline automation and control & lesser employment prospects \\
\hline increased productivity & complexity \\
\hline
\end{tabular}




\section{Conflicts of Interest}

The authors declare no conflict of interest

\section{References}

1. Subbarao, V., Srinivas, K. \& Pavithr, R. S. A survey on internet of things based smart, digital green and intelligent campus. in 2019 4th International Conference on Internet of Things: Smart Innovation and Usages (IoT-SIU) 1-6 (2019).

2. Alrashed, S. Key performance indicators for Smart Campus and Microgrid. Sustainable Cities and Society 60, 102264 (2020).

3. Agdas, D., Srinivasan, R. S., Frost, K. \& Masters, F. J. Energy use assessment of educational buildings: Toward a campus-wide sustainable energy policy. Sustainable Cities and Society 17, 15-21 (2015).

4. Aqeel-ur-Rehman, Abbasi, A. Z. \& Shaikh, Z. A. Building a Smart University Using RFID Technology. in 2008 International Conference on Computer Science and Software Engineering vol. 5 641-644 (2008).

5. Perwej, Dr. Y., Haq, K., Parwej, Dr. F. \& M., M. The Internet of Things (IoT) and its Application Domains. International Journal of Computer Applications 182, 36-49 (2019).

6. Wollschlaeger, M., Sauter, T. \& Jasperneite, J. The Future of Industrial Communication: Automation Networks in the Era of the Internet of Things and Industry 4.0. IEEE Industrial Electronics Magazine 11, 17-27 (2017).

7. Trappey, A. J. C., Trappey, C. V., Govindarajan, U. H., Sun, J. J. \& Chuang, A. C. A Review of Technology Standards and Patent Portfolios for Enabling Cyber-Physical Systems in Advanced Manufacturing. IEEE Access 4, 7356-7382 (2016).

8. Ahlgren, B., Hidell, M. \& Ngai, E. C.-. Internet of Things for Smart Cities: Interoperability and Open Data. IEEE Internet Computing 20, 52-56 (2016).

9. Dlodlo, N., Gcaba, O. \& Smith, A. Internet of things technologies in smart cities. in 2016 ISTAfrica Week Conference 1-7 (2016).

10. Stoyanov, S., Orozova, D. \& Popchev, I. Internet of Things Water Monitoring for a Smart Seaside City. in 2018 20th International Symposium on Electrical Apparatus and Technologies (SIELA) 1-3 (2018).

11. He, H. Research on the Application of Electronic Technology of Internet of Things in Smart City. in 2020 International Conference on Intelligent Transportation, Big Data Smart City (ICITBS) 454-457 (2020).

12. Hossein Motlagh, N., Mohammadrezaei, M., Hunt, J. \& Zakeri, B. Internet of Things (IoT) and the Energy Sector. Energies 13, 494 (2020).

13. Gawali, S. K. \& Deshmukh, M. K. Energy Autonomy in IoT Technologies. Energy Procedia 156, 222-226 (2019).

14. Malche, T. \& Maheshwary, P. Internet of Things (IoT) for building smart home system. in 2017 International Conference on I-SMAC (IoT in Social, Mobile, Analytics and Cloud) (ISMAC) 65-70 (2017).

15. Pătru, I., Carabaş, M., Bărbulescu, M. \& Gheorghe, L. Smart home IoT system. in 2016 15th RoEduNet Conference: Networking in Education and Research 1-6 (2016).

16. Paul, C., Ganesh, A. \& Sunitha, C. An overview of IoT based smart homes. in 2018 2nd International Conference on Inventive Systems and Control (ICISC) 43-46 (2018).

17. Cho, C., Choi, J.-Y., Jeong, J. \& Chung, T.-M. Performance Analysis of Inter-Domain Handoff Scheme Based on Virtual Layer in PMIPv6 Networks for IP-Based Internet of Things. PLoS ONE 12, e0170566 (2017).

18. Purri, S., Choudhury, T., Kashyap, N. \& Kumar, P. Specialization of IoT applications in health care industries. in 2017 International Conference on Big Data Analytics and Computational Intelligence (ICBDAC) 252-256 (2017).

19. Jose, D. V. \& Vijyalakshmi, A. An Overview of Security in Internet of Things. Procedia Computer Science 143, 744-748 (2018). 
20. Sethi, P. \& Sarangi, S. R. Internet of Things: Architectures, Protocols, and Applications. Journal of Electrical and Computer Engineering 2017, 9324035 (2017).

21. Navani, D., Jain, S. \& Nehra, M. S. The Internet of Things (IoT): A Study of Architectural Elements. in 2017 13th International Conference on Signal-Image Technology Internet-Based Systems (SITIS) 473-478 (2017).

22. Zhong, C., Zhu, Z. \& Huang, R. Study on the IOT Architecture and Gateway Technology. in 2015 14th International Symposium on Distributed Computing and Applications for Business Engineering and Science (DCABES) 196-199 (2015).

23. Miao Wu, Ting-Jie Lu, Fei-Yang Ling, Jing Sun \& Hui-Ying Du. Research on the architecture of Internet of Things. in 2010 3rd International Conference on Advanced Computer Theory and Engineering(ICACTE) vol. 5 V5-484-V5-487 (2010).

24. Alansari, Z. et al. Internet of Things: Infrastructure, Architecture, Security and Privacy. in 2018 International Conference on Computing, Electronics Communications Engineering (iCCECE) 150-155 (2018).

25. Xu, X. et al. Research on Key Technologies of Smart Campus Teaching Platform Based on 5G Network. IEEE Access 7, 20664-20675 (2019).

26. Faritha Banu, J., Revathi R., Suganya M., Gladiss Merlin, M.R. IoT based Cloud Integrated Smart Classroom for smart and a sustainable Campus. Procedia Computer Science 172, 77-81 (2020).

27. Zhu, Z. M., Xu, F. Q. \& Gao, X. Research on School Intelligent Classroom Management System Based on Internet of Things. Procedia Computer Science 166, 144-149 (2020). 